\title{
The collection of pre-evacuation times from evacuation trials involving a Hospital Outpatient area and a University Library facility
}

S. Gwynne, E.R. Galea, J. Parke and J. Hickson.

Fire Safety Engineering Group

University of Greenwich, http://fseg.gre.ac.uk

\begin{abstract}
This paper presents data relating to occupant pre-evacuation times from a university and a hospital outpatient facility. Although the two structures are entirely different they do employ relatively similar procedures: members of staff sweeping areas of the structure to encourage individuals to evacuate. However, the manner in which the dependent population reacts to these procedures is quite different. In the hospital case the patients only evacuated once a member of the nursing staff had instructed them to do so while in the university evacuation the students were less dependent upon the actions of the staff with over $50 \%$ of them evacuating with no prior prompting. Although this data may be useful in a variety of areas, it was collected primarily for use within evacuation models.
\end{abstract}

\section{KEYWORDS}

Data collection, Pre-Evacuation Times, Evacuation Trial, Evacuation Modelling

\section{Introduction}

It has until recently been assumed that the most important factor in determining the time taken for an individual to evacuate a structure is the time to cover the distance between their starting location and their eventual point of exit. In the past decade however, through the work of researchers such as Sime [1] and Proulx 2,3], it has become apparent that of equal if not greater importance is the time taken for the occupants to respond to the incident. This response might entail a number of behavioural factors including the perception of the incident, the perception of the seriousness of the incident, the disengagement of the individual from the activity at which they were previously occupied, the collection of goods, the investigation of the incident and finally fleeing from the scene. Generally these behaviours may be categorised as those that occur prior to the instigation of egress movement and that do not directly diminish the time taken to evacuate. All of these activities contribute to what is commonly referred to as the preevacuation time. In many situations the pre-evacuation time may be greater than the actual travel time.

While recent attempts have been made to present and document pre-evacuation times in a variety of settings [4], much work is still required to generate a comprehensive database of pre-evacuation times suitable for fire engineering calculations. This paper describes the collection and analysis of pre-evacuation times from two different types of structure: an educational facility and a hospital. In this paper the pre-evacuation time refers to the time spent by the occupant before starting to evacuate given that the alarm has been raised. The pre-evacuation time is generally dependent on the nature of the occupancy, the state of the occupants, the quality of the management system, the type of alarm system in place, the presence of additional supporting cues (such as the presence of smoke or instructions from a member of staff [5-8]) and even the time of day. Typical 
pre-evacuation times can vary from seconds (occupants are awake, trained, familiar with building, alarm systems and procedures) to many minutes (situations where occupants may require assistance such as in hospitals) [9].

In the past two decades, evacuation modelling has emerged as a valuable means by which to investigate the egress capability of structures in a variety of environments [10]. Initially these models represented little more than an automation of the calculation of regulatory formulations of evacuation. In more recent times, an array of evacuation models have evolved that go much further than this (including CRISP, EXODUS and SIMULEX [11]), endeavouring to represent the evacuation process as accurately as possible. The development of such modelling techniques is highly dependent upon the availability of data [12]. However, the amount and quality of data generally available is quite limited $[2,4,7,13-16]$. The majority of data is collected during evacuation trials (where the purpose of the examination might be the study of the structural performance) or real incidents (where the data is usually based on anecdotal reports). In both cases, the interests of the researchers may not necessarily agree with the requirements of the modellers i.e. the data was not collected with modelling applications in mind. In such cases, modellers are required to use compromised or inappropriate data due to the paucity of data available. The subject of this paper relates to the generation of data-sets concerning the pre-evacuation times of evacuees derived from two unannounced fire drills. In both cases a traditional bell system was used with the support of trained staff. The structures examined are a listed university building and a private hospital. These buildings were selected on the basis of the extensive access provided by the owners as well as the potential usefulness of the data that might be generated. Research staff from the Fire Safety Engineering Group (FSEG) were used to collect the data.

The data has been extracted for several purposes. Firstly to examine the pre-evacuation time distributions generated in these very different buildings and secondly, to analyse the behavioural factors that influenced the pre-evacuation times. The ultimate aim of this work was to use this information to better inform the development and application of evacuation models.

\section{The building layouts}

Two buildings were examined for this work, the Dreadnought building of the University of Greenwich and the Blackheath Private Hospital. The layout of the buildings and the procedures used to collect the data are described below.

\subsection{Dreadnought Building}

\subsubsection{Structure}

The evacuation of the Dreadnought building, located at the University of Greenwich, took place on the $2^{\text {nd }}$ March 2000. The Dreadnought building is a three-storey structure and is currently used for a variety of purposes including providing library services, student computing facilities and a small canteen. Both the ground and first floor house the library services with the computing facilities on the second floor. Lectures are currently held within the computer laboratories on the second floor. The three floors of the building each provide approximately $1000 \mathrm{~m}^{2}$ of usable space. The structure includes six staircases and eight exits. 


\subsubsection{Methodology}

Several methods were used to gather data during the evacuation. A team of 15 FSEG staff was positioned around the exterior of the building to manually record the data. These were used to determine the number of evacuees using particular exits and to determine the overall number of people within the structure. Questionnaires were handed out to the evacuees to examine their experience during the evacuation. Sixty-two closed circuit cameras (permanently located throughout the structure and normally used for security purposes) were also used to gather data relating to the starting location of the evacuees, their behaviour and their pre-evacuation times. A systematic procedure of data extraction was defined with templates being created in order to facilitate a methodical extraction of data from the video footage. This was designed to extract the maximum amount of data from the video evidence in as objective a manner as possible. A large amount of this data was collected relating to the evacuee behaviours exhibited and the factors that influenced their behaviour. The results relating to the primary analysis of the pre-evacuation times and an indication of the secondary analysis of the factors influencing this data are included.

\subsubsection{Population}

The resident population consisted of staff and students. The staff involved were administrative staff, located in offices on the ground floor and were largely segregated from the student population and 'active' staff, consisting of academic and technical staff. This later group were in direct contact with the student population and generally had responsibilities for students during an evacuation. The students were located in the laboratories and in work areas, which may or may not have been in the immediate presence of a member of staff (although there would always have been a member of staff, technical or academic, on the same floor).

In total there were 361 occupants recorded as evacuating during the trial. These were recorded by researchers placed at each of the available points of exit. Only a subset of this population could be used in the analysis of the pre-evacuation times as either their initial locations were not captured by a video camera or their initial locations were captured, but some aspect of their behaviour could not be categorised. In total 247 preevacuation times were recorded from the trial. This included 228 students and 19 members of staff. The university employed a procedure whereby once the alarm sounded nominated members of staff swept each of the rooms, forcing students to leave their work and belongings and inform them of the route that they should adopt. The 'surprise' status of the evacuation was of vital importance to the usefulness of the data set provided (and of course in testing the performance of this procedure), as otherwise members of staff could have been pre-positioned and would also have known that the incident was only a trial and may have acted with less urgency. The structure was occupied relatively recently and so the majority of the students and most of the staff would not have gained much experience with the evacuation procedures.

\subsection{Blackheath Hospital}

\subsubsection{Structure}

The second evacuation drill involved the Blackheath Hospital on the $31^{\text {st }}$ October 2000. The Blackheath Hospital is a 69-bed acute care hospital that serves the private sector. During this trial it was not possible to evacuate inpatients (including bedridden patients), therefore the evacuation was restricted to outpatients and associated staff. Thus none of 
the patients were restricted to beds and the majority of the patients were fully ambulant. Only three members of the senior management were aware that the evacuation was going to occur and had no direct impact on the outcome of the evacuation. The evacuation was therefore 'unannounced'. Standard procedures were employed whereby once the alarm sounded members of staff swept each of the populated areas, informing patients that they had to leave the building for their own safety and the route that they should adopt.

\subsubsection{Methodology}

Eight FSEG members of staff were located around the building, in waiting rooms and corridors to collect the pre-evacuation time and establish the behaviour of the evacuees. Most of the data collectors were out of sight of patients and staff while three data collectors behaved as though they were patients with appointments. Data was recorded manually or using video equipment. One video camera was hidden in a bag while other cameras were in areas out of direct sight from patients and staff. In no case was a camera visible to any potential evacuees. In addition the three observers acting as patients were instructed not to pre-empt evacuee movement or instruction (i.e. not to move before any of the evacuees or artificially delay their movement). In actuality, as is explained later, this was not an issue as patients always sought instruction prior to responding. A degree of cross-referencing between the observations of the researchers was possible to confirm the results produced. There were two classes of occupant that could easily be distinguished: the staff (who had pre-defined roles in case of emergency) and the patients. The pre-evacuation times were only established in three areas of the hospital: the Pathology / Physiotherapy department, a Treatment area and a large Waiting room. These were selected for the expected size and visual access of their populations and for the ability of the researchers to record information without inadvertently affecting the results. The pre-evacuation time and the overall evacuation time of the occupants were recorded. In addition, the evacuees were provided with questionnaires designed to illicit additional information relating to their experiences during the evacuation. In this paper only the preevacuation time data will be referred to. The detail of the analysis for this data-set is not as great as the University data-set, simply due to the quality of the video footage available.

\subsubsection{Population}

The data collected during this examination represented 14 members of staff and 19 patients. These form a subset of the overall hospital population as not all of the hospital was evacuated and not all of the pre-evacuation behaviour of the evacuees could be captured. As such this is a relatively small data set. However, it is indicative of this type of hospital facility. It should be noted that the patient evacuees were all capable of evacuating the structure (i.e. delays in evacuating were not entirely attributable to physical ailments). Given the nature of the structure, members of staff were always located relatively close to the patients included in the trial, although engaged in activities not involving direct interaction with them. Although the hospital required evacuation tests several times a year, given the nature of the hospital and the high turnover of patients, only the staff would have been familiar with the evacuation procedures employed.

\subsection{Operational definition of pre-evacuation times}

The operational definition of pre-evacuation time used in relation to the Dreadnought evacuation was the time taken by an individual to purposefully initiate evacuation. Preevacuation activities included numerous tasks including disengaging from any task at 
which they had previously been occupied, collect coats, break off social activities, etc. All of these types of activities would have contributed to the extent of the overall preevacuation time. Therefore, the time interval from the sounding of the alarm to the point at which the individual initiates their evacuation is the period that defines the preevacuation time for that individual. This type of categorisation was relatively simple for the students, as their behaviour revolved around their own individual response to the incident. The members of staff had other responsibilities requiring them to move around the structure. Therefore great care had to be taken in order to note the initial movement of the staff members rather than a secondary movement.

For the Blackheath data-set, the operational definition of pre-evacuation times for the patients and staff was somewhat different to that just described. For patients, the time recorded reflects the time between the sounding of the alarm and the purposeful evacuation movement (as with the populations during the Dreadnought evacuation). The data recorded for members of staff is the time that they reacted to the call to evacuate within a particular area. This does not preclude the possibility that they had previously acted in an adjacent area. Therefore the data associated with staff members is less rigorously defined, being a superset of pre-evacuation and post-evacuation actions. They are recorded purely to examine their influence upon the behaviour of the patients.

In both cases the pre-evacuation times of the dependent groups (e.g. the students and the patients) are of particular interest and are most easily defined. In the following sections the performance of these groups will be investigated in greater detail. In effect, the staff involved in both evacuations are interesting only in that they execute the procedures and facilitate the evacuation of the dependent population.

\section{The Results}

In this section the analysis of the Dreadnought and the Blackheath data is presented. For each of the analyses performed simple descriptive statistics are presented. In addition, this is supported by graphical evidence relating to the pre-evacuation times of the individual evacuees. Where the data is sufficiently detailed and comprehensive frequency distributions are also provided.

\subsection{Dreadnought Evacuation}

\subsubsection{Pre-evacuation times according to role}

A summary of the overall results are presented in Table 1. On first inspection it appears that there is very little difference between the pre-evacuation times of the staff and students, although a member of staff does respond almost immediately, with the students having pre-evacuation times that were on average only $4.1 \%$ longer. In addition the range of values of the staff and students are similar (see Table 1).

Table 1: Pre-Evacuation time according to occupant type.

\begin{tabular}{|c|c|c|c|}
\hline Occupant Type & $\begin{array}{c}\text { Average (seconds) [Min- } \\
\text { Max] }\end{array}$ & Count & Standard Dev. \\
\hline Staff & $70.8[0-246]$ & 19 & 60.0 \\
\hline Student & $73.7[8-200]$ & 228 & 37.4 \\
\hline
\end{tabular}

However, it is apparent that the data-points representing the staff pre-evacuation times are far more scattered than the student equivalent (see the standard deviation data in Table 1). This dispersal of values is due to the variation in the roles that are expected of staff during an emergency. The pre-evacuation time distributions are compared in Figure 
1(a), where the dispersal of staff pre-evacuation times is apparent. The flatter curve generated by the student data-set indicates the smaller increments in the subsequent preevacuation times. The pre-evacuation time frequency distributions are presented in Figure 1 (b). The curve representing the student data is positively skewed towards a preevacuation time of 60 seconds. The curve representing the staff pre-evacuation times is far more complex, having a multi-modal appearance. This suggests that the staff category itself might have included sub-categories that would have been more useful in representing the data. Secondary analysis was performed in order to investigate this hypothesis.

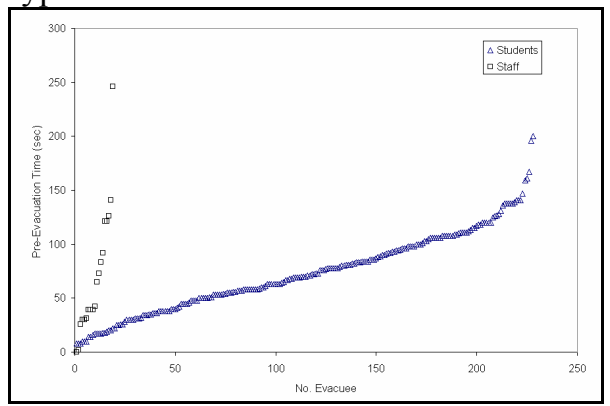

(a)

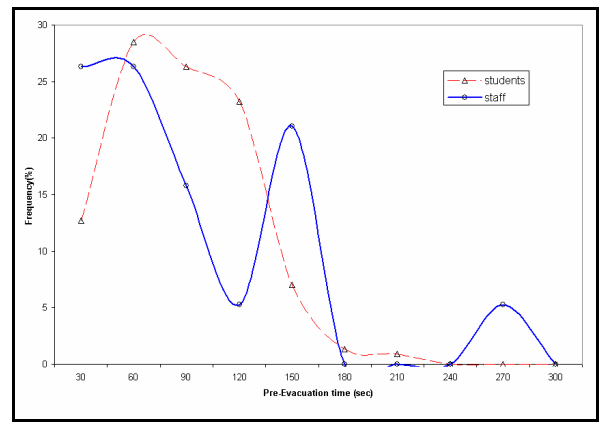

(b)

Figure 1: (a) Pre-evacuation times (b) frequency distribution according to role.

The staff may broadly be categorised into two sub-groupings according to the employment structure: 'active' staff that were moving around the building (either technical or academic staff) and administrative staff who were located in a single office. However, this categorisation did not adequately describe their performance during the evacuation, which was instead controlled by their procedural responsibilities (i.e. whether they had a task to perform or whether they had no prescribed procedural role).

Table 2: Staff Pre-Evacuation times according to employment hierarchy and procedural role

\begin{tabular}{|c|c|c|c|c|c|c|c|}
\hline Job & Avg. & Count & $\begin{array}{c}\text { Stand. } \\
\text { Dev. }\end{array}$ & $\begin{array}{c}\text { Proc. } \\
\text { Role }\end{array}$ & Avg. & Count & $\begin{array}{c}\text { Stand. } \\
\text { Dev. }\end{array}$ \\
\hline Admin. & $\begin{array}{c}67.5 \\
{[26-246]}\end{array}$ & 6 & 87.6 & Role & $\begin{array}{c}84.7 \\
{[0-246]}\end{array}$ & 14 & 64.7 \\
\hline Other & $\begin{array}{c}72.4 \\
{[0-141]}\end{array}$ & 13 & 46.9 & $\begin{array}{c}\text { No } \\
\text { Role }\end{array}$ & $\begin{array}{c}31.8 \\
{[26.0-42]}\end{array}$ & 5 & 60.0 \\
\hline
\end{tabular}

During the evacuation, members of staff may therefore have fulfilled a procedural task (e.g. informed others, locked equipment, etc.), rather than simply being responsible for evacuating as quickly as possible. By examining the pre-evacuation times according to the procedural task adopted, differences are evident (see Table 2). Here a far clearer distinction between the performances of staff members can be observed according to their procedural role, rather than their employment role. A Kruskal-Wallis test was performed to confirm this, comparing the differences in pre-evacuation times according to whether the staff members are differentiated by procedural tasks or the nature of their employment (this test was used as differences in the variance and non-normality were found). The pre-evacuation times were found to be different (at the $5 \%$ level) when the staff were categorised according to their procedural role $(\mathrm{p}=0.04)$, whereas there was no 
significant difference between the sub-groups when the staff were categorised according to their employment role $(\mathrm{p}=0.25)$.

The most important aspect of this analysis is that the staff pre-evacuation distribution can be further refined, according to their role. This refinement provides some explanation for the multi-modal non-normal distribution evident in Figure 1.

\subsubsection{Secondary Analysis of the Student Data-Set}

Secondary analysis was also performed on the student data-set in order to better understand the nature of their response. It was assumed that the differences between the pre-evacuation times generated were not entirely due to physical differences between the individuals or other unsystematic factors but might instead be due to more identifiable factors. This data was therefore examined in the light of this assumption. The analysis of their behaviour was analysed according to a number of factors including the type of location, gender, occupant role, level of isolation, the level of prompting required, the visibility levels of other evacuees, the actions performed prior to evacuating, whether the individual was standing or sitting, etc.. Due to space and time limitations, only the level of prompting required is reported here as being indicative of the type of detailed analysis required (although still representing the full analysis of the impact of this factor). The analysis of the other factors will be included in future publications.

\subsubsection{Level of prompting prior to evacuating}

The level of prompting that occurred prior to the students evacuating was examined to determine whether it had any discernible impact upon the results produced. An individual was assumed to be prompted if they were seen to respond to an obvious act of communication, rather than simply reacting to the sound of the alarm bell. It was assumed prior to the examination that the relationship between the pre-evacuation times and the level of prompting might be relatively complex, possibly depending on a number of other factors. However, as a first analysis this factor was examined independently of any other factors.

Table 3: Pre-Evacuation times according to the level of prompting

\begin{tabular}{|c|c|c|c|c|}
\hline Level of Prompting & Average (seconds) [Min-Max] & Count & Kurtosis & Skewness \\
\hline Without Prompting & $64.8[10-200]$ & 119 & 1.52 & 0.97 \\
\hline Prompted by Student & $91.6[38-196]$ & 22 & 0.6 & 0.92 \\
\hline Prompted by Member of Staff & $81.4[8-147]$ & 87 & -0.88 & -0.35 \\
\hline
\end{tabular}

From Table 3 the average pre-evacuation times appear counter-intuitive, in that the students who were not prompted had both the lowest average pre-evacuation time and the widest range of results. From video analysis and from Figure 2(a), it became apparent that this group included a number of students that were self-motivated (i.e. required no prompting prior to evacuation and had relatively small pre-evacuation times), those that received no prompting (i.e. investigated on their own cognisance) and those that ignored all prompting (i.e. continued working and evacuated when task was completed). This diversity led to the relatively low average pre-evacuation time (largely attributable to the self-motivated evacuees) and a large range of values (the distribution extending from the self-motivated up to those students ignoring all prompting)

From Table 3 and Figure 2 it is apparent that the students that responded to staff prompting had on average lower pre-evacuation times than those being prompted by fellow students. This may have been caused by a number of factors: the prompting of the 
staff being more forceful, the prompting of the staff requiring later reinforcement by the prompting of other students, the prompting of staff being absent therefore leaving the students dependent upon each other to instigate the evacuation process. However there was also a significant group of those responding to members of staff that had preevacuation times of over two minutes. This was due to the late arrival of the member of staff and the dependence of those students upon this arrival. This highlights the dependence of those students awaiting instruction from a member of staff, upon the time of their arrival.

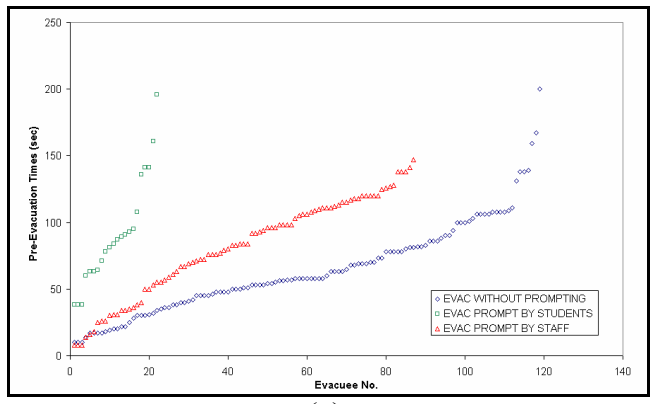

(a)

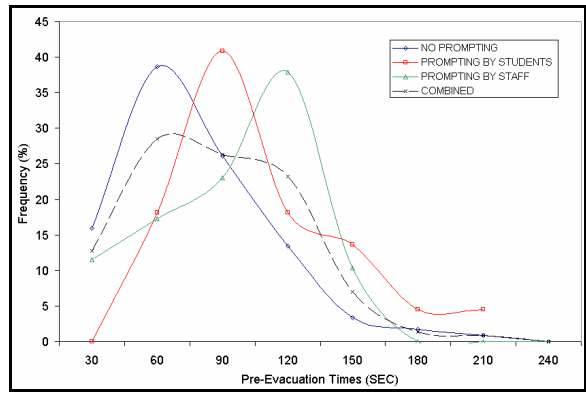

(b)

Figure 2:(a)Pre-evac. times and (b)Frequency distribution according to prompting.

The relationship between the response to prompting and pre-evacuation time is complex. However, it has been demonstrated that a proportion of these students (approximately $50 \%$ ) were willing to evacuate without prompting, whilst the rest required the arrival of a member of staff and/or the cajoling by those around them. This statement implicitly suggests an important relationship with the surrounding population, in both its existence and its willingness to evacuate, as has been suggested in previous work [13]. The application of the current procedure had little impact upon these individuals. Of the student population $38.2 \%$ required staff prompting before they evacuated. Given that they responded to this information, it is possible that these individuals would have been influenced by the more timely arrival of the staff. Finally, $9.6 \%$ of the student population were prompted by other students. It is difficult to comment on what impact the more efficient execution of the current procedure might have had on these individuals. For instance, it might be assumed that the influence of the earlier arrival of members of staff may have had a knock on effect on these individuals.

It is probable that the number of times that prompting took place may have had a cumulative impact on an individual. In addition, the message being communicated will have undoubtedly have had an additional impact. These issues are left for future analysis.

\subsubsection{Use of this data in evacuation modelling}

The utilisation of the descriptive statistics generated in the previous sections would be relatively straight forward within an evacuation model, assuming that the model included a localised representation of pre-evacuation times. Rather than apply a set of preevacuation times across numerous student populations, they may more accurately be able to target the times according to the level of prompting required within that population (possibly reflecting the proximity and actions of members of staff). For each of these categories, a simple range and average has been established, as well as a frequency distribution. The detailed analysis of the data-set allows a more sophisticated 
representation within an evacuation model. Again the aptness of this application will, to a large degree, be dependent upon the inherent sophistication of the model. This type of representation would allow a direct link to be established between the simulated level of engagement and the level of prompting with the eventual pre-evacuation time produced for each of the simulated evacuees.

\subsection{Blackheath Results}

The analysis of these results is far cruder given the smaller data-set and a diminished set of evacuee behaviours. In this section, the pre-evacuation time analysis is categorised according to the areas observed: Pathology and Physiotherapy department, the main Waiting Room and the Treatment area. In each section a sub-set of the observations made by these researchers are presented relating to the pre-evacuation times of the staff and the patients.

\subsubsection{Pathology and Physiotherapy}

In the Pathology and Physiotherapy department only one patient was in the immediate waiting area when the alarm sounded. The video footage shows the patient only started to evacuate on being prompted to do so by a member of staff. Prior to this, two members of staff had concluded that the evacuation was a drill and had not informed the patient to leave. Two other patients responded subsequent to the response of the first patient (see Figure 3(a)). Other members of staff evacuated from adjacent areas of the department, not directly interacting with the patients.

Table 4: Pre-Evacuation Times within Blackheath hospital for Patients / Staff.

\begin{tabular}{|c|c|c|c|c|c|c|}
\hline & \multicolumn{2}{|l|}{ Staff } & \multicolumn{2}{|c|}{ Patients } & \multicolumn{2}{|c|}{ All } \\
\hline Area & Average (sec) & Count & $\begin{array}{c}\text { Average } \\
(\mathrm{sec})\end{array}$ & Count & $\begin{array}{l}\text { Average } \\
(\mathrm{sec})\end{array}$ & Count \\
\hline $\begin{array}{l}\text { Pathology and } \\
\text { Physiotherapy }\end{array}$ & $\begin{array}{c}52.0 \\
{[26.0-91.0]}\end{array}$ & 9 & $\begin{array}{c}37.3 \\
{[30-45]}\end{array}$ & 3 & $\begin{array}{c}48.3 \\
{[26-91]}\end{array}$ & 12 \\
\hline Waiting Room & $\begin{array}{c}26.0 \\
{[16.0-43.0]}\end{array}$ & 4 & $\begin{array}{c}36.3 \\
{[34.0-40.0]}\end{array}$ & 4 & $\begin{array}{c}31.1 \\
{[16.0-43.0]}\end{array}$ & 8 \\
\hline Treatment & $\begin{array}{c}45.0 \\
{[45.0-45.0]}\end{array}$ & 1 & $\begin{array}{c}59.1 \\
{[46.0-66.0]}\end{array}$ & 12 & $\begin{array}{c}58.0 \\
{[45.0-66.0]}\end{array}$ & 13 \\
\hline All Areas & $\begin{array}{c}44.1 \\
{[16.0-19.0]}\end{array}$ & 14 & $\begin{array}{c}50.8 \\
{[30.0-66.0]}\end{array}$ & 19 & $\begin{array}{c}48.0 \\
{[16.0-91.0]}\end{array}$ & 33 \\
\hline
\end{tabular}

The average patient pre-evacuation time was 37.3 seconds, while the average staff preevacuation times was 52.0 seconds. It is apparent that the average staff pre-evacuation time was increased by the late arrival of three members of staff. None of the patients responded prior to being instructed to do so (see Table 4 and Figure 3 (a)).



(a)



(b)

Figure 3: (a) Pre-Evacuation times of the staff/patients in Pathology department (b) Waiting Room. Horizontal line indicates first instruction from member of staff. 


\subsubsection{Waiting Room}

Four patients were initially located in the main Waiting area. The first staff members arrived in the area after 16 seconds (see Figure 3(b)). Instructions were issued to the four patients by a third member of staff after 29 seconds. The final member of staff appeared from another area after the patients had started to evacuate and had no impact on their pre-evacuation times. The ave rage patient pre-evacuation time was 36.3 seconds, while the staff members were seen to act after 26.0 seconds. None of the patients responded prior to being instructed to do so (see Table 4 and Figure 3(b)).

\subsubsection{The Treatment Room}

Six patients were located in the immediate waiting area. Two additional patients were standing in an adjacent corridor while a further 4 patients were located in another (smaller) adjoining waiting room. After 40 seconds the nurse approached the patients, informed them that they would have to leave (she did not inform them what the alarm meant or whether or not the incident was a drill). Once this had been completed, she led the patients upstairs through the main exit collecting other patients en route. This amounted to six other patients who were either missed by other (or this) member of staff or who had arrived after she had begun her sweep.

The average patient pre-evacuation time was 59.1 seconds, while the staff members were seen to act after 45.0 seconds. None of the patients responded prior to being instructed to do so (see Table 4 and Figure 4(a)).

\subsubsection{Use of the Blackheath Results in Modelling}

In the previous sections it became apparent that the behaviour of the staff (nursing staff) had a vital impact on the response of the patients. In all of the three areas observed, the patients only began to evacuate after they had been prompted to do so by a member of staff (see Figure 3 and Figure 4a)). That is not to say that each member of staff prompted patients to leave. What is implied by these events is the importance of the staff and their actions upon the behaviour of the patients.

From Figure 3 and Figure 4(a) a comparison can be made between the pre-evacuation times of the patients and the staff. It should be borne in mind that the reaction of the staff might have included previous actions not recorded in the area under observation. They should therefore be seen as facilitating the response of others rather than having intrinsic value in themselves. From Figure 3 and Figure 4(a) (in conjunction with the observations made during the trial) it is apparent that the behaviour of the patients was entirely dependent upon the actions of the staff. This was supported by the almost complete absence of patient investigative behaviour. This therefore places a huge responsibility on the staff; the patient safety being entirely dependent upon their actions.

To examine the response to the patients more closely a frequency distribution has been generated reflecting the likelihood of a patient responding after a particular time. This was constructed using a class size of 15 seconds, reflecting the relatively limited range of pre-evacuation times of 36 seconds (see Table 4 and Figure 4(b)). It is apparent that the majority of patients responded between 30 and 75 seconds. This reflects the time for staff to instruct them to evacuate and for the patients to collect their baggage, more than the time it took them to process this information or to perform other pre-evacuation behaviour. 


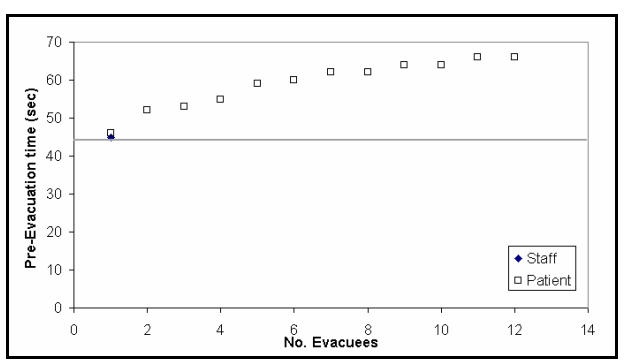

(a)

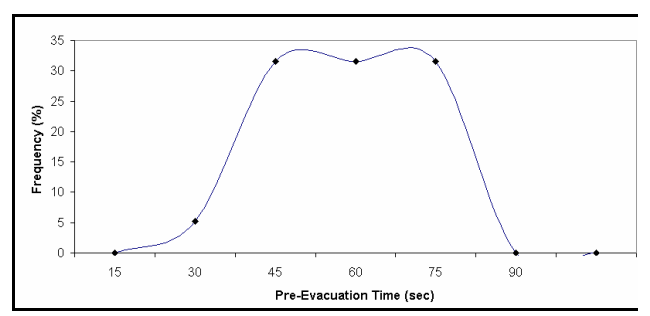

(b)

Figure 4: (a) Pre-Evacuation in the Treatment Room. Horizontal line indicates the first instruction by member of staff. (b) Frequency distribution of patient response.

\section{Conclusions}

This paper has been an attempt to collect and produce data relating to occupant preevacuation times from educational and hospital facilities, primarily for use in evacuation modelling. Although the two structures are entirely different they do employ relatively similar procedures: members of staff sweep areas to encourage individuals to evacuate. However, the manner in which the dependent population reacts to these procedures appears to be structure specific. In the hospital case the patients only evacuated once a member of the nursing staff had instructed them to do so, indicating the importance of the staff actions upon the safe egress of the patients (shaped through the design of the procedure). It is expected that this type of relationship would predominate in similar hospital facilities and be accentuated by additional physical dependencies in less ambulant patients. In contrast during the university evacuation the students were less dependent upon the actions of the staff with over $50 \%$ of them evacuating with no prior prompting. This has important consequences in the design of procedures for this type of structure. It is expected that the Dreadnought facility is not atypical of this type of teaching establishment.

It should be borne in mind that, in addition to the procedural actions employed, the relationship that the dependent populations have with the structure and the staff present will have an impact on their response. In the university, the students may utilise the structure without necessarily being dependent upon the staff. Within a hospital setting this will not usually be the case. In addition, the fact that the students will be far more familiar with the 'normal' non-evacuation environment within the structure - as they are located in the structure as part of their daily routine-will influence their behaviour during less familiar situations such as evacuation. This is reflected in the dependency relationships exhibited during the trial. The impact of familiarity on evacuation behaviour has been previously established $[1,3,10]$

There is an inherent difficulty in using experimental data within an evacuation model as for the most part the context of this data is often lost or ignored. Generally, when trial data is reported it tends to focus on the results produced rather than the factors that may have influenced these results. As such they tend to provide guidance as to the expected ranges of data rather than the nature of the factors that may have influenced the distributions. As evacuation models become more sensitive to a variety of pre-evacuation conditions, so they require more detailed data, which is accompanied by increasingly 
comprehensive contextual information. If the fire engineering community is to improve its modelling capabilities, more attention must therefore be paid to the factors that influence the resulting behaviour, rather than simply quantifying the behaviour observed.

\section{Acknowledgements}

The authors are indebted to the management staff of the University of Greenwich and Blackheath Hospital for allowing the use of their facilities for this work. Professor Galea is indebted to the CAA for their financial support of his personal chair in Mathematical Modelling.

\section{References}

1 Sime, J., "Human Behaviour In Fires Summary Report", CFBAC Report No.450, Portsmouth Polytechnic, 1992.

2 Proulx, G., "Time Delay To Start Evacuating Upon Hearing The Fire Alarm", Proceedings Of Human Factors And Ergonomics Society 38th Annual Meeting , pp811-815, 1994.

3 Proulx, G., 'Lessons Learned On Occupants' Movement Times And Behaviour During Evacuation Drills", Interflam 96, pp1007-1011, 1996.

4 Fahy, R and Proulx, G., "Toward Creating a Database on Delay Times to Start Evacuation and Walking Speeds For use in Evacuation Modelling", $2^{\text {nd }}$ International Symposium on Human Behaviour in Fire, pp175-185, M.I.T.,USA, Interscience (London), 2001.

5 Geyer,T., Bellamy, L., Max-Lino, R., Harrison,P., Bahrami, Z. And Modha, B., "An Evaluation Of The Effectiveness Of The Components Of Information Fire Warning Systems", In Sime J.,(Ed) Safety In The Built Environment, E And Fn Spon, pp36-47, 1988.

6 Bellamy, L.J, Geyer, T.A.W., Max-Lino, R., Harrison, P.I., Bahrami, Z. And Modha, B.,"An Evaluation Of The Effectiveness Of The Components Of Informative Fire Warning Systems", Safety In The Built Environment, Ed:Sime,J., pp36-47,Spon, ISBN 0-419-14480-3,1988.

7 Proulx, G. And Fahy,R., "A Study Of The New York World Trade Centre Evacuation", Proceedings Of The Third International Symposium On Fire Safety Science, Eds: Cox, G. And Langford, G., pp199 -209, Elsevier, London, 1991

8 Proulx, G.,Sime, J.,"To Prevent Panic In An Underground Emergency: Why Not Tell People The Truth?", Fire Safety Science - 3rd Symposium, Elsevier, Appl. Sci., NY, pp843-853,1991.

9 Fire Safety Engineering in buildings, Part 1 Guide to the application of fire safety engineering principles, DD240: Part 1: 1997, Publisher: BSI., ISBN 058027952 9, 1997.

10 Gwynne S., Galea, E. R., Lawrence, P.J. and Filippidis, L., "Modelling Occupant Interaction with Fire Conditions Using the buildingEXODUS model",Fire Safety Journal,36,pp327-357, 2001.

11 Gwynne, S., Galea, E., R., Owen, M. Lawrence, P. J. and Filippidis, L.," "Revie w of modelling methodologies used in the simulation of evacuation", Journal of Building and the Environment, 34, pp441-749, 1999.

12 Weckman, H., Lehtimaki, S. And Mannikko, S., "Evacuation Of A Theatre: Exercise Vs. Calculations", Proceedings Of The 1st International Symposium On Human Behaviour In Fire, Shield,J (Ed), Fire Engineering Research And Technology Centre, University Of Ulster, ISBN 1859231039, pp479-488, 1998.

13 Horasan, M and Bruck, D., "Investigation Of A Behavioural Response Model For Fire Emergency Situations In Secondary Schools", Proceedings Of Fourth International Symposium On Fire Safety Science, Ed: Kashiwagi, T.L, pp715-726, ISBN 1-886279-00-4, 1994.

14 Olsson, P., and Regan, M.,A.,"A Comparison Between Actual and Predicted Evacuation Times",Human Behaviour in Fire, Proceedings of the First International Symposium, Shield, J. (Ed), Fire Engineering Research And Technology Centre, University Of Ulster, ISBN 1859231039, pp461-469, 1998.

15 Lerup, L., Cronrath, D., Liu, J.K.C, "Fires In Nursing Facilities", Fires And Human Behaviour (2nd Edition), Ed. D.Canter, Fulton, pp155-180, 1990

16 Butler, G.W., "Private Communication - Observation Of A Routine Fire Drill At A Multi Occupied Building In Newcastle Upon Tyne", 23rd April 1993. 